\title{
Construction of a parametrix for the Cauchy problem of some weakly hyperbolic equation III
}

\author{
By Atsushi Yoshikawa \\ (Received November 1, 1976)
}

\section{§ 0. Introduction}

Let

$(0.0)$

$$
\begin{aligned}
P= & P\left(t, x, D_{t}, D_{x}\right)=D_{t}^{2}-t^{2} \sum_{j, k=1}^{n} a_{j k}(t, x) D_{j} D_{k}+ \\
& +b_{0}(t, x) D_{t}+\sum_{j=1}^{n} b_{j}(t, x) D_{j}+c(t, x) .
\end{aligned}
$$

Here $a_{j k}(t, x), b_{l}(t, x), c(t, x)$ are $C^{\infty}$ functions of $(t, x)=\left(t, x_{1}, \cdots, x_{n}\right) \in \boldsymbol{R} \times$ $\times \boldsymbol{R}^{n}$, and

$$
D_{t}=-i \partial / \partial t, \quad D_{j}=-i \partial / \partial x_{j}, \quad j=1, \cdots, n,
$$

$i^{2}=-1$, as usual. We assume that

(0.1) $\quad a_{j k}(t, x)=a_{k j}(t, x), j, k=1, \cdots, n$, be real valued,

(0.2) $\quad a_{j k}(t, x)=\delta_{j k} \quad$ for $\quad t \geqq t_{0}, \quad|x| \geqq r_{0}$,

$t_{0}, r_{0}$ being some positive numbers, and

$$
\sum_{j, k=1}^{n} a_{j k}(t, x) \xi_{j} \xi_{k} \geqq c \sum_{j=1}^{n} \xi_{j}^{2}
$$

for $(t, x) \in \boldsymbol{R}^{n+1}, \xi=\left(\xi_{1}, \cdots, \xi_{n}\right) \in \boldsymbol{R}^{n}, c$ being a positive constant independent of $t, x, \xi$.

Consider the following Cauchy problem:

$$
\begin{aligned}
& P v(t, x)=0, \quad t>0, \quad x \in \boldsymbol{R}^{n}, \\
& v(\tau, x)=f(x), \quad D_{t} v(\tau, x)=g(x), \quad x \in \boldsymbol{R}^{n} .
\end{aligned}
$$

Here $\tau \geqq 0$ and $f, g$ are given distributions in $\mathcal{E}^{\prime}\left(\boldsymbol{R}^{n}\right)$.

In our previous paper [2], we have constructed good parametrices (cf. [2], Definition 0.1) for the problem (0.4) (0.5) when $a_{j k}(t, x) \equiv \delta_{j k}$. The purpose of the present paper is to extend our previous reasoning to cover the operator of the form $(0.0)$ with $(0.1) \sim(0.3)$. Note that the assumption $(0.2)$ is just to avoid unnecessary complications and in fact is not restrictive at all.

Before stating our main result, we introduce several notations. 
Let

$$
a(t, x, \xi)=\left(\sum_{j, k=1}^{n} a_{j k}(t, x) \xi_{j} \xi_{k}\right)^{1 / 2}
$$

for $(t, x, \xi) \in \boldsymbol{R} \times \boldsymbol{R}^{n} \times \boldsymbol{R}^{n} \backslash 0$. Thus $a(t, x, \xi)$ is positive and positively homogeneous of degree 1 in the variables $\xi$. Assume as before [1], [2] that

$$
\operatorname{Im} \sum_{j=1}^{n} b_{j}(0, x) \xi_{j} \quad \text { be uniformly bounded }
$$

for $x \in \boldsymbol{R}^{n}$ and $\xi$ on the unit sphere $\boldsymbol{S}^{n-1}$. Then we set

$$
m(\sigma)=-\frac{1}{4}+\frac{1}{4} \sup \left\{\sigma \operatorname{Im} \sum_{j=1}^{n} b_{j}(0, x) \xi_{j} / a(0, x, \xi)\right\}, \sigma^{2}=1,
$$

the superimum being taken over $(x, \xi) \in \boldsymbol{R}^{n} \times \boldsymbol{S}^{n-1}$. Finally, let $\boldsymbol{\phi}^{\sigma}(t, \tau, x, \xi)$, $\sigma^{2}=1$, be respectively solutions of the following equations :

$$
\phi_{t}^{\sigma}=\sigma t a\left(t, x, \phi_{x}^{\sigma}\right), \quad \sigma^{2}=1,
$$

with

(0.10)

$$
\left.\phi^{\sigma}\right|_{t=\tau}=\langle x, \xi\rangle, \quad(\tau \geqq 0) .
$$

To abovid unnecessary complications, we assume that $\phi^{\sigma}(t, \tau, x, \xi), \sigma^{2}=1$, be well-defined everywhere in the large (cf. (0.2)).

Now our main result reads as follows.

THEOREM. There exist symbols

(0.11) $\quad p_{c}(t, \tau, x, \xi) \in S^{\varsigma, 2 m(\sigma)+\varsigma, 2 m(-\sigma)+1+\varsigma}$,

(0. 12) $\quad q_{\sigma}(t, \tau, x, \xi) \in S^{\varepsilon-1,2 m(\sigma)+\varepsilon, 2 m(-\sigma)+\varepsilon}$,

(0.13) $\quad \tilde{p}_{\sigma}(t, \tau, x, \xi) \in S_{+}^{\varepsilon, 2 m(-\sigma)+1+\varepsilon}$,

(0.14) $\quad \tilde{q}_{\sigma}(t, \tau, x, \xi) \in S_{+}^{\varepsilon-1,2 m(-\sigma)+\bullet}$,

(0. 15) $\quad p_{\sigma}^{\#}(t, \tau, x, \xi) \in S_{\infty}^{\varepsilon}$,

(0. 16) $\quad q_{\sigma}^{\sharp}(t, \tau, x, \xi) \in S_{\infty}^{\varepsilon-1}$,

$\sigma^{2}=1$, such that

$$
\begin{aligned}
& {\left[E_{0}(t, \tau) f\right](x)=} \\
& =\sum_{\sigma= \pm 1}(2 \pi)^{-n} \iint e^{i\left\{\phi^{\sigma}(t, \tau, x, \xi)-\langle y, \xi\rangle\right\}} p_{\sigma}(t, \tau, x, \xi) f(y) d y d \xi+ \\
& \quad+\sum_{\sigma= \pm 1}(2 \pi)^{-n} \iint e^{i\left(\phi^{\sigma}(t, \tau, x, \xi)-\langle y, \xi\rangle\right\}} \widetilde{p}_{\sigma}(t, \tau, x, \xi) f(y) d y d \xi+ \\
& \quad+\sum_{\sigma= \pm 1}(2 \pi)^{-n} \iint e^{i\left\langle\phi^{\sigma}(t, \tau, x, \xi)-\langle y, \xi\rangle\right\}} p_{\sigma}^{\sharp}(t, \tau, x, \xi) f(y) d y d \xi
\end{aligned}
$$

and 
(U. 18)

$$
\begin{aligned}
& {\left[E_{1}(t, \tau) g\right](x)=} \\
& =\sum_{\sigma= \pm 1}(2 \pi)^{-n} \iint e^{i\left[\hat{\sigma}^{\sigma}(t, \tau, x, \xi)-\langle y, \xi\rangle\right]} q_{o}(t, \tau, x, \xi) g(y) d y d \xi+ \\
& +\sum_{\sigma= \pm 1}(2 \pi)^{-n} \iint e^{\left.i \mid \phi^{\sigma}(t, \tau, x, \xi)-\langle y, \xi\rangle\right)} \tilde{q}_{\sigma}(t, \tau, x, \xi) g(y) d y d \xi+ \\
& +\sum_{\sigma= \pm 1}(2 \pi)^{-n} \iint e^{i\left(\phi^{\sigma}(t, \tau, x, \xi)-\langle y, \xi\rangle\right)} q_{\sigma}^{\sharp}(t, \tau, x, \xi) g(y) d y d \xi
\end{aligned}
$$

form a good parametrix for the problew (0.4) (0.5). Here the integrals in (0.17) and (0.18) are oscillatory ones over $\boldsymbol{R}^{n} \times \boldsymbol{R}^{n}$. The symbol classes in (0.11) (0.16) are given in [2]. $\varepsilon$ is an arbitrary positive number and may be omitted if $n=1$ and $b_{1}(0, x) / a_{11}(0, x)$ is independent of $x$.

The method to be employed is essentially the same as before [1], [2]. However, in our previous discussions we have largely relied on the simple form of the partial differential operator in consideration. In the present paper, a certain novelty appears in the construction of $p_{\sigma}(t, \tau, x, \xi)$ and $q_{o}(t, \tau, x, \xi)$. Though the basic ibea of using semi-homogeneous decomposition is common to [1], [2], we need several modifications of our previous arguments. The most important improvement in our present discussions is that we discuss directly the transport operators

$$
T^{\sigma}=e^{-i \phi^{\sigma}} P e^{i \phi^{\circ}}, \quad \sigma^{2}=1 .
$$

We give two decompositions of the transport operators $T^{o}$, the semi-homogeneous one and the homogeneous one. Using the former decomposition, we construct $p_{\sigma}(t, \tau, x, \xi)$ and $q_{\sigma}(t, \tau, x, \xi)$. Although the underlying techniques employed in this stage is common to [1], [2], the present treatment makes clearer the meaning of $p_{\sigma}(t, \tau, x, \xi)$ and $q_{\sigma}(t, \tau, x, \xi)$. The remaining constructions of $\widetilde{p}_{\sigma}(t, \tau, x, \xi), \tilde{q}_{\sigma}(t, \tau, x, \xi), p_{\sigma}^{\sharp}(t, \tau, x, \xi), q_{\sigma}^{\sharp}(t, \tau, x, \xi)$ are done by the homogeneous decompositions of the transport operators and are thus just the same as before [2].

The content of the present paper is as follows:

$\S 1$. Transport operators and their decompositions;

$\S 2$. Constructions of $p_{\sigma}(t, \tau, x, \xi)$ and $q_{o}(t, \tau, x, \xi), \sigma^{2}=1$;

$\S 3$. End of the proof of Theorem.

\section{$\S 1$. Transport operators and their decompositions.}

Let $\tilde{x}=(t, x)$ and $\tilde{\xi}=\left(\xi_{0}, \xi\right), x, \xi \in \boldsymbol{R}^{n}$. We set

$$
\begin{aligned}
& P_{2}(\widetilde{x}, \tilde{\xi})=\xi_{0}^{2}-t^{2} \sum_{j, k=1}^{n} a_{j k}(t, x) \xi_{j} \xi_{k}, \\
& P_{1}(\widetilde{x}, \tilde{\xi})=b_{0}(t, x) \xi_{0}+\sum_{j=1}^{n} b_{j}(t, x) \xi_{j},
\end{aligned}
$$




$$
\begin{aligned}
& P_{2}(\tilde{x}, \widetilde{D})=D_{t}^{2}-t^{2} \sum_{j, k=1}^{n} a_{j k}(t, x) D_{j} D_{k}, \\
& P_{1}(\widetilde{x}, \widetilde{D})=b_{0}(t, x) D_{t}+\sum_{j=1}^{n} b_{j}(t, x) D_{j}, \\
& P_{0}(\widetilde{x}, \widetilde{D})=c(t, x) .
\end{aligned}
$$

Then

$$
P=P(\widetilde{x}, \widetilde{D})=P_{2}(\widetilde{x}, \widetilde{D})+P_{1}(\widetilde{x}, \widetilde{D})+P_{0}(\widetilde{x}, \widetilde{D}) .
$$

As usual we write

$$
\begin{aligned}
& P_{2}^{(k)}(\tilde{x}, \tilde{\xi})=\partial P_{2}(\widetilde{x}, \tilde{\xi}) / \partial \xi_{k}, \\
& P_{2(l)}(\widetilde{x}, \tilde{\xi})=\partial P_{2}(\widetilde{x}, \tilde{\xi}) / \partial x_{l},
\end{aligned}
$$

for $k, l=0,1, \cdots, n,\left(x_{0}=t\right)$. The transport operators $T^{s}, \sigma^{2}=1$, are then expressed in the following way:

$$
\begin{aligned}
T & =e^{-i \phi^{\sigma}} P e^{i \phi^{\sigma}} \\
& =\sum_{j=0}^{n} P_{2}^{(j)}\left(\widetilde{x}, \phi_{\tilde{x}}^{\sigma}\right) D_{j}+i P_{2}(\widetilde{x}, \widetilde{D}) \phi^{\sigma}+P_{1}\left(\widetilde{x}, \phi_{\tilde{x}}^{\sigma}\right)+P(\widetilde{x}, \widetilde{D}),
\end{aligned}
$$

where $\phi^{\sigma}, \sigma^{2}=1$, are given by $(0.9)(0.10)$ and $D_{0}=D_{t}, \phi_{\tilde{x}}^{o}=\left(\phi_{t}^{o}, \phi_{x}^{\sigma}\right)$.

The transport operators $T^{\sigma}, \sigma^{2}=1$, admit semi-homogeneous decompositions. Namely, we have the following

PROPOSIrION 1.1. The transport operators $T^{\sigma}, \sigma^{2}=1$, are decomposed into the following formal sums:

$$
T^{o}=\sum_{k=0}^{\infty} T_{k}^{o},
$$

$T_{k}^{o}$ being semi-homogeneous of degree $1-k / 2, k=0,1,2, \cdots$. More precisely, for each $k$,

$$
T_{k}^{o}=\sum_{l=0}^{k} \tau^{l} T_{k, l}^{o}
$$

$T_{k, l}^{o}$ being partial differential operators independent of $\tau$ and semi-homogeneous of degree $1-k / 2+l / 2$; namely,

$$
\begin{aligned}
T_{0}^{o} & =T_{0,0}^{o}= \\
& =D_{t}^{2}+2 \sigma t a(0, x, \xi) D_{t}+\sum_{j=1}^{n} b_{j}(0, x) \xi_{j}-i \sigma a(0, x, \xi), \\
T_{k, 0}^{o} & =t^{k}|\xi| C(x, \xi)+t^{k-2}\left\{C(x, \xi) t D_{t}+C\left(x, \xi ; D_{x}\right)\right\}
\end{aligned}
$$

and

$$
T_{k, l}^{o}=t^{k-l}|\xi|\left\{C(x, \xi)+C(x, \xi) t D_{t}+C\left(x, \xi ; D_{x}\right)\right\}
$$

for $k=1,2, \cdots ; l=1,2, \cdots, k$. Here $C(x, \xi)$ denote various suitable functions in $(x, \xi) \in \boldsymbol{R}^{n} \times \boldsymbol{R}^{n} \backslash 0$ of homogeneous of degree zero, and $C\left(x, \xi ; D_{x}\right)$ various 
suitable partial differential operators in $x$ with coefficients smooth functions of $(x, \xi) \in \boldsymbol{R}^{n} \times \boldsymbol{R}^{n} \backslash 0$ of homogeneous of degree zero. In (1.13), the second term in the right hand side disappears when $k=1$.

Proof. The proposition follows from (1.9) and the Taylor expansions around $(t, \tau)=(0,0)$ of $P_{2}^{(j)}\left(\widetilde{x}, \phi_{\tilde{x}}^{\sigma}\right), j=0, \cdots, n, P_{2}(\widetilde{x}, \widetilde{D}) \phi^{\sigma}, P_{1}\left(\widetilde{x}, \phi_{\tilde{x}}^{\sigma}\right)$ and the coefficients of $P(\widetilde{x}, \widetilde{D})$.

REMARK 1.2. In our previous paper [2], due to the simple form of the operator $P$ for that case, the terms corresponding to $T_{k, l}^{a}, l \geqq 1$, $(k=1,2, \cdots)$, did not appear. The presence of these terms causes some new complexity. This complexity can be handled in the frame of our previous discussions with a little modification as will be shown in the next section.

We end this section by the following obvious proposition.

Proposirion 1.3. The transport operators $T^{o}, \sigma^{2}=1$, admit the following decompositions in homogeneous parts:

$$
\begin{aligned}
& T^{o}=\widetilde{T}_{1}^{o}+\widetilde{T}_{0}^{o}, \\
& \widetilde{T}_{1}^{a}=\sum_{j=0}^{n} P_{2}^{(j)}\left(\widetilde{x}, \phi_{\tilde{x}}^{\sigma}\right) D_{j}+i P_{2}(\widetilde{x}, \widetilde{D}) \phi^{\sigma}+P_{1}\left(\widetilde{x}, \phi_{\tilde{x}}^{\sigma}\right), \\
& \widetilde{T}_{0}^{o}=P(\widetilde{x}, \widetilde{D}) .
\end{aligned}
$$

$\widetilde{T}_{j}^{o}, j=0,1$, are homogeneous of degree $j$, respectively.

§2. Constructions of $p_{\sigma}(t, \tau, x, \xi)$ and $q_{\sigma}(t, \tau, x, \xi), \sigma^{2}=1$.

We begin by the following observation.

Proposition 2.1. Let $p(\tau, x, \xi)$ and $q(\tau, x, \xi)$ be given. Then the solutions $u_{\sigma}(t, \tau, x, \xi), \sigma^{2}=1$, of the equations.

$$
T_{0}^{o} u_{s}=0, \quad \sigma^{2}=1,
$$

with the initial conditions

$$
\begin{aligned}
& \left.\sum_{\sigma= \pm 1} u_{\sigma}\right|_{t=\tau}=p(\tau, x, \xi), \\
& \left.\sum_{\sigma= \pm 1}\left\{\sigma \tau a(0, x, \xi)+D_{t}\right\} u_{\sigma}\right|_{t=\tau}=q(\tau, x, \xi)
\end{aligned}
$$

are given by

$$
\begin{aligned}
& u_{\sigma}(t, \tau, x, \xi)=-\frac{\sigma i}{2} \psi_{o}\left(a(0, x, \xi)^{1 / 2} t, B\right) \times \\
& \quad \times\left[p ( \tau , x , \xi ) \left\{i \sigma a(0, x, \xi)^{1 / 2} \tau \psi_{-\sigma}\left(a(0, x, \xi)^{1 / 2} \tau, B\right)-\right.\right.
\end{aligned}
$$




$$
\begin{aligned}
& \left.-\psi_{-\sigma}^{\prime}\left(a(0, x, \xi)^{1 / 2} \tau, B\right)\right\}+ \\
& \left.+i a(0, x, \xi)^{-1 / 2} q(\tau, x, \xi) \phi_{-\sigma}\left(a(0, x, \xi)^{1 / 2} \tau, B\right)\right],
\end{aligned}
$$

where

$$
B=B(x, \xi)=\sum_{j=1}^{n} b_{j}(0, x) \xi_{j} / a(0, x, \xi)
$$

and $\psi_{\sigma}, \sigma^{2}=1$, are the entire functions defined by Proposition 2.1 in [1].

PROOF. This is an immediate consequence of Corollary 2.4 in [1].

Now let us set formally

$$
p_{\sigma}(t, \tau, x, \xi) \sim \sum_{j=0}^{\infty} \sum_{m=0}^{\infty} p_{j \sigma}^{m}(t, \tau, x, \xi)
$$

and

$$
q_{v}(t, \tau, x, \xi) \sim \sum_{j=0}^{\infty} \sum_{m=0}^{\infty} q_{j o}^{m}(t, \tau, x, \xi) .
$$

Here $p_{j \sigma}^{m}(t, \tau, x, \xi)$ and $q_{j \sigma}^{m}(t, \tau, x, \xi)$ are respectively semihomogeneous of degree $-(m+j) / 2$ and $-(m+j+1) / 2$. Namely,

$$
p_{j \sigma}^{m}\left(\lambda^{-1 / 2} t, \lambda^{-1 / 2} \tau, x, \lambda \xi\right)=\lambda^{-(m+j) / 2} p_{j \sigma}^{m}(t, \tau, x, \xi)
$$

and

$$
q_{j \sigma}^{m}\left(\lambda^{-1 / 2} t, \lambda^{-1 / 2} \tau, x, \lambda\right)=\lambda^{-(m+j+1) / 2} q_{j \sigma}^{m}(t, \tau, x, \xi)
$$

for $\lambda>0$.

We determine $p_{j \sigma}^{m}$ and $q_{j \sigma}^{m}$ by the following equations (Compare with the discussions in [2] before Proposition 2.2).

For $m=0$, we solve $p_{0 \sigma}^{0}$ from the equations

$$
T_{0}^{o} p_{0 \sigma}^{0}=0, \quad \sigma^{2}=1,
$$

with the initial conditions

$$
\begin{aligned}
& \left.\sum_{\sigma= \pm 1} p_{0 \sigma}^{0}\right|_{t=\tau}=1, \\
& \left.\sum_{\sigma= \pm 1}\left\{\sigma t a(0, x, \xi)+D_{t}\right\} p_{0 \sigma}^{0}\right|_{t=\tau}=0 .
\end{aligned}
$$

Then we determine $p_{j a}^{0}, j \geqq 1$, from the equations

$$
T_{0}^{o} p_{j \sigma}^{0}=-\sum_{k=1}^{j} T_{k, 0}^{o} p_{j-k \sigma}^{0}, \quad \sigma^{2}=1 .
$$

This is done as before [2] via the asymptotic expansion of the right hand side in the sector $S=\{t \in C \backslash 0 ;|\arg t|<\pi / 4\}$. For each $j$ and $\sigma$, we then have a formal series solution of (2.13) and we can thus specify $p_{j \sigma}^{0}$ as the solution, entire in $t$, which has this formal solution as the asymptotic expansion in the sector $S$. 
For $m \geqq 1$, we solve $p_{0 o}^{m}$ from the equations

$$
T_{0}^{o} p_{0 o}^{m}=-\sum_{k=1}^{m} \tau^{k} T_{k, k}^{o} p_{0 s}^{m-k}
$$

with the initial conditions

$$
\begin{aligned}
& \left.\sum_{\sigma= \pm 1} p_{0 \sigma}^{m}\right|_{t=\tau}=-\left.\sum_{j=1}^{m} p_{j o}^{m-j}\right|_{t=\tau}, \\
& \begin{aligned}
\sum_{\sigma= \pm 1} & \left.\left\{\sigma t a(0, x, \xi)+D_{t}\right\} p_{o \sigma}^{m}\right|_{t=\tau}= \\
& =-\left.\sum_{\sigma= \pm 1}\left\{\sigma t a(0, x, \xi)+D_{t}\right\} \sum_{j=1}^{m} p_{j o}^{m-j}\right|_{t=\tau}- \\
& -\left.\sum_{\sigma= \pm 1} \sigma \sum_{k=1}^{m} \tau^{k+1}|\xi| c_{k}^{\sigma}(x, \xi) \sum_{j=0}^{m-k} p_{m-j-k \sigma}^{j}\right|_{t=\tau} .
\end{aligned}
\end{aligned}
$$

Here $c_{k}^{\sigma}(x, \xi), k=1,2, \cdots$, are functions of $(x, \xi) \in \boldsymbol{R}^{n} \times \boldsymbol{R}^{n} \backslash 0$, homogeneous of degree zero, and determined by the Taylor expansion of $\phi_{t}^{\tau}(\tau, \tau, x, \xi)$ around $\tau=0$ :

$$
\begin{gathered}
\phi_{t}^{o}(\tau, \tau, x, \xi)=\sum_{k=0}^{\infty} \sigma \tau^{k+1}|\xi| c_{k}^{o}(x, \xi), \quad \sigma^{2}=1, \\
|\xi| c_{0}^{o}(x, \xi)=a(0, x, \xi) .
\end{gathered}
$$

We then determine $p_{j o}^{m}, j \geqq 1$, from the equations

$$
T_{0}^{o} p_{j \sigma}^{m}=-\sum_{k=1}^{j} T_{k, 0}^{o} p_{j-k \sigma}^{m}-\sum_{l=1}^{m} \sum_{k=0}^{j} \tau^{l} T_{k+l, l}^{o} p_{j-k \sigma}^{m-l}
$$

as the solutions, entire in $t$, with the prescribed asymptotic beeaviors in the sector $S$.

In a similar manner, we solve $q_{0 \sigma}^{0}$ from the equations

$$
T_{0}^{s} q_{0 \sigma}^{0}=0, \quad \sigma^{2}=1,
$$

with the initial conditions

$$
\left.\sum_{\sigma= \pm 1} q_{0 \sigma}^{0}\right|_{t=\tau}=0 \text {, }
$$

$$
\left.\sum_{\sigma= \pm 1}\left\{\sigma t a(0, x, \xi)+D_{t}\right\} q_{0 \sigma}^{0}\right|_{t=\tau}=1 \text {. }
$$

We then determine $q_{j a}^{0}, j \geqq 1$, from the equations

$$
T_{0}^{o} q_{j \sigma}^{0}=-\sum_{k=1}^{j} T_{k, 0}^{o} q_{j-k \sigma}^{0}, \quad \sigma^{2}=1,
$$

as the solutions, entire in $t$, with the prescribed asymptotic behaviors in the sector $S$.

For $m \geqq 1$, we solve $q_{0 s}^{m}$ from the equations

$$
T_{\sigma}^{0} q_{0_{\sigma}}^{m}=-\sum_{k=1}^{m} \tau^{k} T_{k, k}^{\sigma} q_{0 o}^{m-k}
$$

with the initial conditions

$$
\left.\sum_{o= \pm 1} q_{0 o}^{m}\right|_{t=\tau}=-\left.\sum_{j=1}^{m} q_{j \sigma}^{m-j}\right|_{t=\tau},
$$




$$
\begin{aligned}
\sum_{\sigma= \pm 1} & \left.\left\{\sigma t a(0, x, \xi)+D_{t}\right\} q_{0 \sigma}^{m}\right|_{t=\tau}= \\
= & -\left.\sum_{\sigma= \pm 1}\left\{\sigma t a(0, x, \xi)+D_{t}\right\} \sum_{j=1}^{m} q_{j \sigma}^{m-j}\right|_{t=\tau}- \\
& -\left.\sum_{\sigma= \pm 1} \sigma \sum_{k=1}^{m} \tau^{k+1}|\xi| c_{k}^{\sigma}(x, \xi) \sum_{j=0}^{m-k} q_{m-k-j \sigma}^{j}\right|_{t=\tau} .
\end{aligned}
$$

We finally determine $q_{j \sigma}^{m}, j \geqq 1$, from the equations

$$
\begin{aligned}
T_{0}^{o} q_{j o}^{m}= & -\sum_{k=1}^{j} T_{k, 0}^{o} q_{j-k \sigma}^{m}- \\
& -\sum_{l=1}^{m} \sum_{k=0}^{j} \tau^{l} T_{k+l, l}^{o} q_{j-k \sigma}^{m-l}
\end{aligned}
$$

as the solutions, entire in $t$, with the prescribed asymptotic behaviors in the sector $S$.

We can thus recurrently determine all the $p_{j o}^{m}$ and $q_{j o}^{m}$. The growth estimates of $p_{j o}^{m}$ and $q_{j o}^{m}$ are given by the following

Proposition 2.2. For $|\xi|=1$,

$$
\begin{aligned}
& p_{j \sigma}^{m}(t, \tau, x, \xi)= \\
& \quad=C t^{-2 \tilde{\mu}_{\sigma}+j}(\log t)^{N}\left(1+o_{t}(1)\right) \tau^{-2 \tilde{\mu}_{-\sigma}+m+1}(\log \tau)^{M}\left(1+o_{\tau}(1)\right),
\end{aligned}
$$

(2. 30) $\quad q_{j o}^{m}(\tau, \tau, x, \xi)=C \tau^{j+m-1}(\log \tau)^{M}\left(1+o_{\tau}(1)\right)$

as $t, \tau \rightarrow \infty$ in the sector $S$. Here

$$
\tilde{\mu}_{\sigma}=\frac{1}{4}+\sigma \frac{i}{4} B(x, \xi), \quad \sigma^{2}=1,
$$

$B(x, \xi)$ being that of (2.5), and $C$ stands for various functions of $x, \xi$ appropriate to each case, $M, N$ various non-negative integers. $o_{t}(1)$ and $o_{\tau}(1)$ denote various functions of $t$ and $\tau$, respectively, in $O^{-1+\varepsilon}\left(S, \boldsymbol{R}^{n} \times \boldsymbol{S}^{n-1}\right), \varepsilon>0$.

PROOF. This is proved essentially in the same way as Proposition 2.3 of [2]. The apparition of $M, N$ in the present case is due to the forms of $T_{k, l}^{o}$ (cf. (1.14), in particular).

In view of the semi-homogeneity requirements on $p_{j o}^{m}$ and $q_{j o}^{m}$, we can interpret the above result in terms of the symbol classes in [2]. Thus we obtain

Corollary 2.3. For any $m$ and $j$, we have 
(2. 32) $\quad p_{j o}^{m}(t, \tau, x, \xi) \in S^{s, 2 m(\sigma)+j+\varepsilon, 2 m(-\sigma)+m+1+\varepsilon}$,

(2. 33) $\quad q_{j \sigma}^{m}(t, \tau, x, \xi) \in S^{c-1,2 m(\sigma)+j+c, 2 m(-\sigma)+m+\varepsilon}$,

(2. 34) $\quad p_{j \sigma}^{m}(\tau, \tau, x, \xi) \in S^{\mathrm{c}, 2 m(\sigma)+2 m(-\sigma)+m+j+1+2 \varepsilon}$,

(2. 35) $\quad q_{j \sigma}^{m}(\tau, \tau, x, \xi) \in S^{\varepsilon-1,2 m(\sigma)+2 m(-\sigma)+m+j+2 \varepsilon}$,

$\sigma^{2}=1$. Here $\varepsilon$ is an arbitrary positive number and

$$
m(\sigma)=-\frac{1}{4}+\frac{1}{4} \sup \left\{\sigma \operatorname{Im} \sum_{j=1}^{n} b_{j}(0, x) \xi_{j} / a(0, x, \xi)\right\}
$$

as defined by (0.8).

Now let us apply Proposition 1.8 and Corollary 1.9 of [2] to $p_{j \sigma}^{m}$ and $q_{j \sigma}^{m}$. First we have

$$
p_{o}^{m}(t, \tau, x, \xi) \in S^{c, 2 m(\sigma)+\varsigma, 2 m(-\sigma)+m+1+\varepsilon}
$$

and

$$
q_{\sigma}^{m}(t, \tau, x, \xi) \in S^{\varepsilon-1,2 m(\sigma)+\varepsilon, 2 m(-\sigma)+m+\varepsilon},
$$

$\sigma^{2}=1$, such that, for all $N>0$,

$$
p_{\sigma}^{m}-\sum_{j<N} p_{j \sigma}^{m} \in S^{\varepsilon, 2 m(\sigma)+N+\varepsilon, 2 m(-\sigma)+m+1+\varepsilon}
$$

and

$$
q_{o}^{m}-\sum_{j<N} q_{j \sigma}^{m} \in S^{\varepsilon-1,2 m(\sigma)+N+\varepsilon, 2 m(-\sigma)+m+\varepsilon} .
$$

We then have

$$
p_{\sigma}(t, \tau, x, \xi) \in S^{\varepsilon, 2 m(\sigma)+\varepsilon, 2 m(-\sigma)+1+\varepsilon}
$$

and

(2. 41) $\quad q_{\sigma}(t, \tau, x, \xi) \in S^{\varepsilon-1,2 m(\sigma)+\varsigma, 2 m(-\sigma)+\varepsilon}$, $\sigma^{2}=1$, such that, for all $N>0$,

(2. 42) $\quad p_{\boldsymbol{\sigma}}-\sum_{m<N} p_{\sigma}^{m} \in S^{\varsigma, 2 m(\sigma)+\varepsilon, 2 m(-\sigma)+N+1+\varsigma}$

and

$$
q_{\sigma}-\sum_{m<N} q_{\sigma}^{m} \in S^{\varepsilon-1,2 m(\sigma)+\varepsilon, 2 m(-\sigma)+N+\varepsilon} .
$$

Let us set

$$
p_{00}(\tau, x, \xi)=\sum_{\sigma= \pm 1} p_{\sigma}(\tau, \tau, x, \xi)-1
$$

$$
q_{00}(\tau, x, \xi)=\sum_{\sigma= \pm 1} q_{\sigma}(\tau, \tau, x, \xi) \text {, }
$$

$$
p_{01}(\tau, x, \xi)=\left.\sum_{\sigma= \pm 1}\left\{\phi_{t}^{\sigma}(t, \tau, x, \xi)+D_{t}\right\} p_{\sigma}(t, \tau, x, \xi)\right|_{t=\tau},
$$




$$
q_{01}(\tau, x, \xi)=\left.\sum_{\sigma= \pm 1}\left\{\phi_{t}^{\sigma}(t, \tau, x, \xi)+D_{t}\right\} q_{\sigma}(t, \tau, x, \xi)\right|_{t=\tau}-1
$$

Then we have the following

Proposition 2. 4

(2. 48) $\quad p_{00}(\tau, x, \xi) \in S^{\iota, \infty}$,

(2.49) $\quad q_{00}(\tau, x, \xi) \in S^{\tau-1, \infty}$,

(2.50) $\quad p_{01}(\tau, x, \xi) \in S^{c+1, \infty}$,

(2.51) $\quad q_{01}(\tau, x, \xi) \in S^{c, \infty}$.

Proof. We prove (2.48) and (2.50). (2.49) and (2.51) can be shown analogously. For any $M>0$,

$$
\begin{aligned}
& p_{00}(\tau, x, \xi)=\left\{\sum_{\sigma= \pm 1} \sum_{m=1}^{M} \sum_{j=0}^{M} p_{j \sigma}^{m}(\tau, \tau, x, \xi)-1\right\}+ \\
& \quad+\sum_{o= \pm 1} \sum_{m=0}^{M}\left\{p_{\sigma}^{m}(\tau, \tau, x, \xi)-\sum_{j=0}^{M} p_{j \sigma}^{m}(\tau, \tau, x, \xi)\right\}+ \\
& \quad+\sum_{\sigma= \pm 1}\left\{p_{\sigma}(\tau, \tau, x, \xi)-\sum_{m=0}^{M} p_{\sigma}^{m}(\tau, \tau, x, \xi)\right\} .
\end{aligned}
$$

The last two sums belong to $S^{s, 2 m(\sigma)+2 m(-\sigma)+M+2+2 t}$ by virtue of $(2.38)$ and (2. 42). Now by (2.11) and (2.15), the first term reduces to

$$
\sum_{\sigma= \pm 1} \sum_{m+j>M} p_{j a}^{m}(\tau, \tau, x, \xi),
$$

which also belongs to $S^{(, 2 m(\sigma)+2 m(-\sigma)+M+2+2 \epsilon}$ because of (2.32). This proves (2.48). On the other hand, for any $M>0$,

$$
\begin{aligned}
p_{01}(\tau, x, \xi) & = \\
=\left.\sum_{\sigma= \pm 1}\left\{\phi_{t}^{\sigma}(t, \tau, x, \xi)+D_{t}\right\}\left(p_{\sigma}-\sum_{m=0}^{M} p_{\sigma}^{m}\right)\right|_{t=\tau}+ & \\
& +\left.\sum_{\sigma= \pm 1}\left\{\phi_{t}^{\sigma}(t, \tau, x, \xi)+D_{t}\right\} \sum_{m=0}^{M}\left(p_{\sigma}^{m}-\sum_{j=0}^{M} p_{j o}^{m}\right)\right|_{t=\tau}+ \\
& +\left.\sum_{\sigma= \pm 1}\left\{\phi_{t}^{\sigma}(t, \tau, x, \xi)+D_{t}\right\} \sum_{j, m=0}^{M} p_{j o}^{m}\right|_{t=\tau} .
\end{aligned}
$$

The first two sums belong to $S^{c+1,2 m(\sigma)+2 m(-\sigma)+M+1+2 c}$ in view of (2.38) and (2. 42). Now by the Taylor expansion,

$$
\begin{aligned}
\phi_{t}^{\sigma}(\tau, \tau, x, \xi)=\sigma \tau a(0, x, \xi) & +\sigma \sum_{k=1}^{M} \tau^{k+1}|\xi| c_{k}^{o}(x, \xi)+ \\
& +\sigma \tau^{M+2}|\xi| c_{M+1}^{o}(\tau, x, \xi),
\end{aligned}
$$

$c_{M+1}^{o}(\tau, x, \xi)$ being homogeneous of degree zero. Then by (2.12) and (2.16),

$$
\begin{aligned}
& \left.\sum_{\sigma= \pm 1}\left\{\phi_{t}^{\sigma}(t, \tau, x, \xi)+D_{t}\right\} \sum_{j, m=0}^{M} p_{j o}^{m}\right|_{t=\tau}= \\
& \quad=\left.\sum_{\sigma= \pm 1} \sigma \tau^{M+2}|\xi| c_{M+1}^{o}(\tau, x, \xi) \sum_{j, m=0}^{M} p_{j o}^{m}\right|_{t=\tau}+
\end{aligned}
$$




$$
\begin{aligned}
& +\left.\sum_{\sigma= \pm 1}\left\{\sigma t a(0, x, \xi)+D_{t}\right\} \sum_{j+m>M} p_{j \sigma}^{m}\right|_{t=\tau}+ \\
& +\left.\sum_{\sigma= \pm 1} \sigma \sum_{k=1}^{M} \tau^{k+1}|\xi| c_{k}^{\sigma}\left(x, \xi^{*}\right) \sum_{j+m+k>M} p_{j \sigma}^{m}\right|_{t=\tau}
\end{aligned}
$$

belongs to $S^{c+1,2 m(\sigma)+m(-\sigma)+M+1+2 c}$. This proves $(2.50)$.

Let us set, for $\sigma^{2}=1$,

$$
r_{\sigma}(t, \tau, x, \xi)=T^{o} p_{\sigma}(t, \tau, x, \xi)
$$

and

$$
s_{\sigma}(t, \tau, x, \xi)=T^{o} q_{\sigma}(t, \tau, x, \xi) .
$$

Then we have the following proposition, which has no analogue in our previous discussions [2].

Proposition 2.5. For $\sigma^{2}=1$,

$$
r_{\sigma}(t, \tau, x, \xi) \in S^{c+1,2 m(\sigma)+\imath, 2 m(-\sigma)+1+\varepsilon}
$$

and

$$
s_{o}(t, \tau, x, \xi) \in S^{\iota, 2 m(\sigma)+\iota, 2 m(-\sigma)+\triangleleft} .
$$

Furthermore, for any $N, J>0$, there exist $r_{\sigma, N, J}(t, \tau, x, \xi)$ and $s_{\sigma, N, J}(t, \tau, x, \xi)$ such that

$$
\begin{aligned}
& r_{\sigma, N, J}(t, \tau, x, \xi) \in S^{\iota+1, J+1,2 m(-\sigma)+1+\iota}, \\
& r_{o}-r_{\sigma, N, J} \in S^{\iota+1,2 m(\sigma)+\iota, 2 m(-\sigma)+N+2+\iota}
\end{aligned}
$$

and

$$
s_{\sigma, N, J}(t, \tau, x, \xi) \in S^{\iota, J+1,2 m(-\sigma)+\iota},
$$

$$
\boldsymbol{s}_{\sigma}-\boldsymbol{S}_{\sigma, N, J} \in S^{\mathrm{c}, 2 m(\sigma)+\iota, 2 m(-\sigma)+N+1+\triangleleft} .
$$

Proof. (2.54) and (2.55) follow from (2.40) and (2.41) in view of Propositions 1.5, 1.6 of [2] and Proposition 1.3. We prove (2.56) and (2.57). (2.58) and (2.59) are shown analogously.

Let

$$
p_{o, N}=\sum_{m=0}^{N} p_{o}^{m} .
$$

Then by (2.42) and Propositions 1.5, 1.6 of [2],

$$
T^{\sigma}\left(p_{\sigma}-p_{\sigma, N}\right) \in S^{\iota+1,2 m(\sigma)+\imath, 2 m(-\sigma)+2+N+\varsigma} .
$$

Let us show

$$
T^{o} p_{\sigma, N} \in S^{c+1, \infty, 2 m(-\sigma)+1+\triangleleft}
$$

modulo $S^{c+1,2 m(\sigma)+\iota, 2 m(-\sigma)+2+N+c}$. 
Let $J>0$ and

$$
T^{j}=\sum_{k=0}^{\infty} \sum_{l=0}^{\infty} \tau^{l} \hat{T}_{k, l}^{o} .
$$

Here $\hat{T}_{k, l}^{o}=T_{k, l}^{o}$ for $l=0, \cdots, k ; k=0, \cdots, j+2 N+1$ and $\hat{T}_{k, l}^{o}=0$ for $k \geqq J+$ $2 N+3 . \quad \hat{T}_{j+2 N+2, l}{ }^{o}, l=0, \cdots, J+2 N+2$, correspond to the remainder terms in the Taylor expansion of $T^{\sigma}$ so that

$$
\hat{T}_{J+2 N+2,0}^{\sigma}=t^{J+2 N+2}|\xi| C\left(t, \tau, x, \xi, D_{x}\right)+t^{J+2 N} C\left(t, \tau, x, \xi, t D_{t}, D_{x}\right)
$$

and

$$
\hat{T}_{J+2 N+2, l}^{\sigma}=t^{J+2 N+2-l}|\xi| C\left(t, \tau, x, \xi, t D_{t}, D_{x}\right),
$$

$l=1, \cdots, J+2 N+2, C\left(t, \tau, x, \xi, t D_{t}, D_{x}\right)$ being suitable partial differential operators in $t D_{t}, D_{x}$ with coefficients homogeneous of degree zero. Let

$$
\begin{aligned}
& \hat{p}_{j o}^{m}=p_{j o}^{m} \text { for } j=0, \cdots, J+m ; m=0, \cdots, N, \\
& \hat{p}_{j o}^{m}=0 \text { for } j>J+m+1 \text { if } m \leqq N \text { or for any } j \text { if } m \geqq N+1,
\end{aligned}
$$

and

$$
\hat{p}_{j+m+1 \sigma}^{m}=p_{\sigma}^{m}-\sum_{j<J+m+1} p_{j \sigma}^{m}, m=0, \cdots, N .
$$

Then $p_{\sigma, N}=\sum_{m=0}^{N} \sum_{j=0}^{\infty} \hat{p}_{j \sigma}^{m}$ and

$$
\begin{aligned}
& T^{\sigma} p_{o, N}= \sum_{m=0}^{\infty} \sum_{j=m}^{\infty} \sum_{l=0}^{m} \sum_{k=0}^{j-m} \tau^{l} \hat{T}_{j-m-k+l, l}^{o} \hat{p}_{k \sigma}^{m-l}= \\
&=\sum_{m=0}^{N} \sum_{j=m}^{J+m} \sum_{l=0}^{m} \sum_{k=0}^{j-m} \tau^{l} \hat{T}_{j-m-k+l, l}^{\sigma} p_{k \sigma}^{m-l}+ \\
& \quad+\sum_{m=0}^{N} \sum_{j=J+m+1}^{\infty} \sum_{l=0}^{m} \sum_{k=0}^{j-m} \tau^{l} \hat{T}_{j-m-k+l, l}^{\sigma} \hat{p}_{k \sigma}^{m-l}+ \\
& \quad+\sum_{m=N+1}^{\infty} \sum_{j=0}^{\infty} \sum_{l=0}^{m} \sum_{k=0}^{j-m} \tau^{l} \hat{T}_{j-m-k+l, l}^{o} \hat{p}_{k \sigma}^{m-l} .
\end{aligned}
$$

The first sum vanishes by virtue of (2.10), (2.13), (2.14) and (2.18). The second sum belongs to $S^{1+\varepsilon, 2 m(\sigma)+J+1+\varepsilon, 2 m(-\sigma)+1+\imath}$ since

$$
\tau^{\imath} \hat{T}_{j-m-k+l, l}^{\sigma} \hat{p}_{k \sigma}^{m-l} \in S^{1+\varepsilon, 2 m(\sigma)+j-m+\varepsilon, 2 m(-\sigma)+m+1+\triangleleft}
$$

by means of (1.12), (1.13), (1.14), (2.32), (2.38), (2.62) and (2.63). Similarly, the last sum, which is finite, belongs to $S^{1+\varepsilon, 2 m(\sigma)+\varepsilon, 2 m(-\sigma)+N+2+\sigma}$. Therefore, for any $J$,

$$
T^{\sigma} p_{\sigma, N} \in S^{1+\varepsilon, 2 m(\sigma)+J+1+\varepsilon, 2 m(-\sigma)+1+\varsigma}
$$

modulo a term in $S^{1+\iota, 2 m(\theta)+\iota, 2 m(-\sigma)+N+2+\bullet}$. This shows (2.61). (2.56) and (2.57) now result from (2.60) and (2.61) by setting

$$
r_{\sigma, N, J}=T^{o} p_{\sigma, N}-\sum_{m=N+1}^{\infty} \sum_{j=J+m+1}^{\infty} \sum_{l=0}^{m} \sum_{k=0}^{j-m} \tau^{l} \hat{T}_{j-m-k+l, l}^{o} \hat{p}_{k \sigma}^{m-l} .
$$

From (2.56), (2.57) and (2.58) (2.59), we have the following

Corollary 2. 6. There exist symbols $r_{o}^{0}(t, \tau, x, \xi), r_{o}^{1}(t, \tau, x, \xi), r_{o}^{2}(t, \tau, x, \xi)$ 
and $s_{\sigma}^{0}(t, \tau, x, \xi), s_{\sigma}^{1}(t, \tau, x, \xi), s_{\sigma}^{2}(t, \tau, x, \xi), \sigma^{2}=1$, such that

(2. 64) $\quad r_{o}(t, \tau, x, \xi)=\sum_{j=0}^{2} r_{0}^{j}(t, \tau, x, \xi)$,

(2. 65) $\quad s_{\sigma}(t, \tau, x, \xi)=\sum_{j=0}^{2} s_{\sigma}^{j}(t, \tau, x, \xi)$

with

(2. 66) $\quad r_{\sigma}^{0}(t, \tau, x, \xi) \in S^{\iota+1, \infty, 2 m(-\sigma)+1+\triangleleft}$,

(2. 67) $\quad s_{\sigma}^{0}(t, \tau, x, \xi) \in S^{c, \infty, 2 m(-\sigma)+1+\iota}$,

(2.68) $\quad r_{\sigma}^{1}(t, \tau, x, \xi) \in S_{\infty}^{1+\varepsilon}$,

(2. 69) $s_{\sigma}^{1}(t, \tau, x, \xi) \in S_{\infty}^{\mathbf{s}}$,

(2. 70) $\quad r_{\sigma}^{2}(t, \tau, x, \xi), s_{\sigma}^{2}(t, \tau, x, \xi) \in S_{+}^{-\infty}$.

Proof. This follows immediately from the definitions of the symbol classes. In fact, $S^{\mu, r, \infty} \subset S_{\infty}^{\mu}$.

\section{§3. End of the proof of Theorem.}

In view of (2.66) and (2.67) we may assume that

$$
\begin{aligned}
& r_{\sigma}^{0}(t, \tau, x, \xi) \in S_{+}^{c+1,2 m(-\sigma)+1+\_}, \quad \sigma^{2}=1, \\
& s_{\sigma}^{0}(t, \tau, x, \xi) \in S_{+}^{c, 2 m(-\sigma)+\iota}, \quad \sigma^{2}=1,
\end{aligned}
$$

and

$$
r_{\sigma}^{0}(t, \tau, x, \xi) \text { and } s_{\sigma}^{0}(t, \tau, x, \xi) \text { be flat at } t=0 .
$$

Then we have, just as before [1] [2], the following

Proposition 3. 1. There exist symbols

$$
\begin{aligned}
& \widetilde{p}_{\sigma}(t, \tau, x, \xi) \in S_{+}^{\varepsilon, 2 m(-\sigma)+1+\triangleleft}, \\
& \widetilde{q}_{\sigma}(t, \tau, x, \xi) \in S_{+}^{\varepsilon-1,2 m(-\sigma)+\varepsilon},
\end{aligned}
$$

$\sigma^{2}=1$, such that

$$
\begin{aligned}
& T^{\sigma} \widetilde{p}_{\sigma}(t, \tau, x, \xi)+r_{\sigma}^{0}(t, \tau, x, \xi) \in S_{+}^{-\infty}, \\
& T^{o} \tilde{q}_{\sigma}(t, \tau, x, \xi)+s_{\sigma}^{0}(t, \tau, x, \xi) \in S_{+}^{-\infty}, \\
& \widetilde{p}_{\sigma}(t, \tau, x, \xi) \text { and } \tilde{q}_{\sigma}(t, \tau, x, \xi) \text { are flat at } t=0 .
\end{aligned}
$$

Proof. This can be shown in the same way as $\S 3$ of [2] in view of Proposition 1.3.

Let us set 
(3. 9) $\quad \widetilde{p}_{00}(\tau, x, \xi)=\sum_{o= \pm 1} \widetilde{p}_{\sigma}(\tau, \tau, x, \xi)$,

(3. 10) $\quad \tilde{q}_{00}(\tau, x, \xi)=\sum_{\sigma= \pm 1} \tilde{q}_{\sigma}(\tau, \tau, x, \xi)$,

(3.11) $\quad \widetilde{p}_{01}(\tau, x, \xi)=\left.\sum_{o= \pm 1}\left\{\boldsymbol{\phi}_{t}^{\sigma}(t, \tau, x, \xi)+D_{t}\right\} \widetilde{p}_{\sigma}(t, \tau, x, \xi)\right|_{t=\tau}$,

(3.12) $\quad \tilde{q}_{01}(\tau, x, \xi)=\left.\sum_{\sigma= \pm 1}\left\{\phi_{t}^{q}(t, \tau, x, \xi)+D_{t}\right\} \tilde{q}_{\sigma}(t, \tau, x, \xi)\right|_{t=\tau}$.

Then Proposition 3. 1 immediately implies the following

Corollary 3. 2.

(3. 13) $\tilde{p}_{00}(\tau, x, \xi) \in S^{c, \infty}$,

(3.14) $\quad \tilde{q}_{00}(\tau, x, \xi) \in S^{-1, \infty}$,

(3. 15) $\tilde{p}_{01}(\tau, x, \xi) \in S^{\iota+1, \infty}$,

(3. 16) $\quad \tilde{q}_{01}(\tau, x, \xi) \in S^{c, \infty}$.

Now set

(3.17) $\quad p_{0}(\tau, x, \xi)=-p_{00}(\tau, x, \xi)-\widetilde{p}_{00}(\tau, x, \xi)$,

(3. 18) $\quad q_{0}(\tau, x, \xi)=-q_{00}(\tau, x, \xi)-\tilde{q}_{00}(\tau, x, \xi)$,

(3. 19) $\quad p_{1}(\tau, x, \xi)=-p_{01}(\tau, x, \xi)-\widetilde{p}_{01}(\tau, x, \xi)$,

(3.20) $\quad q_{1}(\tau, x, \xi)=-q_{01}(\tau, x, \xi)-\tilde{q}_{01}(\tau, x, \xi)$.

The following result is then immediate from Proposition 2.4 and Corollary 3.2.

Corollary 3. 3.

(3. 21) $\quad p_{0}(\tau, x, \xi) \in S^{\iota, \infty}$,

(3. 22) $\quad q_{0}(\tau, x, \xi) \in S^{-1, \infty}$,

(3.23) $p_{1}(\tau, x, \xi) \in S^{\star+1, \infty}$,

(3. 24) $\quad q_{1}(\tau, x, \xi) \in S^{c, \infty}$.

Then just in the same way as the proofs of Propositions 4.3 and 4.4 of [2], we have the following

Proposition 3.4. There exist symbols

(3. 25) $\quad p_{o}^{\sharp}(t, \tau, x, \xi) \in S_{\infty}^{\text {c }}$

and

(3. 26) $\quad q_{\sigma}^{\sharp}(t, \tau, x, \xi) \in S_{\infty}^{\mathrm{c}-1}$, 
$\sigma^{2}=1$, such that

(3. 27) $\quad T^{\sigma} p_{a}^{\sharp}(t, \tau, x, \xi)+r_{\sigma}^{1}(t, \tau, x, \xi) \in S_{\infty}^{-\infty}$,

(3. 28) $\quad T^{o} q_{\sigma}^{\#}(t, \tau, x, \xi)+s_{\sigma}^{1}(t, \tau, x, \xi) \in S_{\infty}^{-\infty}$

and

(3. 29) $\quad \sum_{\sigma= \pm 1} p_{\sigma}^{\#}(\tau, \tau, x, \xi)-p_{0}(\tau, x, \xi) \in S^{-\infty}$,

(3. 30) $\left.\quad \sum_{\sigma= \pm 1}\left\{\phi_{t}^{\sigma}(t, \tau, x, \xi)+D_{t}\right\} p_{\sigma}^{\#}(t, \tau, x, \xi)\right|_{t=\tau}-p_{1}(\tau, x, \xi) \in S^{-\infty}$,

(3. 31) $\quad \sum_{\sigma= \pm 1} q_{\sigma}^{\sharp}(\tau, \tau, x, \xi)-q_{0}(\tau, x, \xi) \in S^{-\infty}$,

(3. 32) $\left.\quad \sum_{o= \pm 1}\left\{\phi_{t}^{\gamma}(t, \tau, x, \xi)+D_{t}\right\} q_{\sigma}^{\sharp}(t, \tau, x, \xi)\right|_{t=\tau}-q_{1}(\tau, x, \xi) \in S^{-\infty}$.

The proof of Theorem is now complete.

\section{References}

[1] Yoshikawa, A.: Construction of a parametrix for the Cauchy problem of some weakly hyperbolic equation I, Hokkaido Math. J., 6 (1977), 313-344.

[2] Yoshikawa, A.: Construction of a parametrix for the Cauchy problem of some weakly hyperbolic equation II, Hokkaido Math. J., 7 (1978), 1-26.

Department of Mathematics Hokkaido University 